\title{
ITERATED ABSOLUTE VALUES OF DIFFERENCES OF CONSECUTIVE PRIMES
}

\author{
ANDREW M. ODLYZKO
}

Dedicated to the memory of D. H. Lehmer

\begin{abstract}
Let $d_{0}(n)=p_{n}$, the $n$th prime, for $n \geq 1$, and let $d_{k+1}(n)=$ $\left|d_{k}(n)-d_{k}(n+1)\right|$ for $k \geq 0, n \geq 1$. A well-known conjecture, usually ascribed to Gilbreath but actually due to Proth in the 19th century, says that $d_{k}(1)=1$ for all $k \geq 1$. This paper reports on a computation that verified this conjecture for $k \leq \pi\left(10^{13}\right) \approx 3 \times 10^{11}$. It also discusses the evidence and the heuristics about this conjecture. It is very likely that similar conjectures are also valid for many other integer sequences.
\end{abstract}

\section{INTRODUCTION}

Let $p_{1}=2, p_{2}=3, \ldots$ be the primes in their natural ordering, and set

$$
\begin{gathered}
\quad d_{0}(n)=p_{n}, \quad n \geq 1, \\
d_{k+1}(n)=\left|d_{k}(n)-d_{k}(n+1)\right|, \quad k \geq 0, n \geq 1 .
\end{gathered}
$$

Table 1 (next page) shows $d_{k}(n)$ for $0 \leq k \leq 20,1 \leq n \leq 20$. Note that $d_{k}(1)=1$ for $1 \leq k \leq 20$. As was pointed out by $\mathrm{H}$. C. Williams, Proth [15] claimed to prove that $d_{k}(1)=1$ for all $k \geq 1$, but his proof was faulty. More recently, Gilbreath (unpublished) independently conjectured that $d_{k}(1)=1$ for all $k \geq 1$. (See Problem A10 in [7], and also [8].) This is usually referred to as Gilbreath's conjecture.

Gilbreath's conjecture was verified for $k \leq 63,419$, that is for all primes $<792,731$, by Killgrove and Ralston [8], who were fellow students of Gilbreath at UCLA in the late $1950 \mathrm{~s}$. This paper reports on a verification of this conjecture for all primes $<10^{13}$, so that $d_{k}(1)=1$ for $1 \leq k \lesssim 3.4 \times 10^{11}$. The computational results are presented in $\S 3$, and the algorithms that were used are described in $\S 4$.

For a general sequence $d_{0}(n)$, to compute $d_{k}(1)$ it is necessary to compute $d_{j}(i)$ for all $i+j \leq k+1$, so that for $k \sim 3.4 \times 10^{11}$ approximately $5 \times 10^{22}$ numbers have to be computed, far too many for the technology of today or the near future. The computations for $d_{0}(n)=p_{n}$ were possible because of special properties of the primes. Note that $d_{k}(1)$ is odd and $d_{k}(2), d_{k}(3), \ldots$, are even for all $k \geq 1$. If for some $N$ we find a $K$ such that $d_{K}(1)=1$ while $d_{K}(n)=0$ or 2 for all $1 \leq n \leq N$, then we can conclude that $d_{k}(1)=1$ for $K \leq k \leq N+K-1$. Let $G(N)$ denote the minimal $k$ (if it exists) such that $d_{j}(1)=1$ for $1 \leq j \leq k$ and $d_{k}(n)=0$ or 2 for $1 \leq n \leq N$. Computations

Received by the editor July $15,1992$.

1991 Mathematics Subject Classification. Primary 11N05, 11Y99; Secondary 11K36, 11Y16, $68 \mathrm{Q} 25$. 
TABLE 1. Iterated differences $d_{k}(n)$ for $0 \leq k \leq 20,1 \leq n \leq 20$

\begin{tabular}{|c|c|c|c|c|c|c|c|c|c|c|c|c|c|c|c|c|c|c|c|c|}
\hline$k \backslash n$ & 1 & 2 & 3 & 4 & 5 & 6 & 7 & 8 & 9 & 10 & 11 & 12 & 13 & 14 & 15 & 16 & 17 & 18 & 19 & 20 \\
\hline 0 & 2 & 3 & 5 & 7 & 11 & 13 & 17 & 19 & 23 & 29 & 31 & 37 & 41 & 43 & 47 & 53 & 59 & 61 & 67 & 71 \\
\hline 1 & 1 & 2 & 2 & 4 & 2 & 4 & 2 & 4 & 6 & 2 & 6 & 4 & 2 & 4 & 6 & 6 & 2 & 6 & 4 & 2 \\
\hline 2 & 1 & 0 & 2 & 2 & 2 & 2 & 2 & 2 & 4 & 4 & 2 & 2 & 2 & 2 & 0 & 4 & 4 & 2 & 2 & 4 \\
\hline 3 & 1 & 2 & 0 & 0 & 0 & 0 & 0 & 2 & 0 & 2 & 0 & 0 & 0 & 2 & 4 & 0 & 2 & 0 & 2 & 2 \\
\hline 4 & 1 & 2 & 0 & 0 & 0 & 0 & 2 & 2 & 2 & 2 & 0 & 0 & 2 & 2 & 4 & 2 & 2 & 2 & 0 & 2 \\
\hline 5 & 1 & 2 & 0 & 0 & 0 & 2 & 0 & 0 & 0 & 2 & 0 & 2 & 0 & 2 & 2 & 0 & 0 & 2 & 2 & 2 \\
\hline 6 & 1 & 2 & 0 & 0 & 2 & 2 & 0 & 0 & 2 & 2 & 2 & 2 & 2 & 0 & 2 & 0 & 2 & 0 & 0 & 0 \\
\hline 7 & 1 & 2 & 0 & 2 & 0 & 2 & 0 & 2 & 0 & 0 & 0 & 0 & 2 & 2 & 2 & 2 & 2 & 0 & 0 & 0 \\
\hline 8 & 1 & 2 & 2 & 2 & 2 & 2 & 2 & 2 & 0 & 0 & 0 & 2 & 0 & 0 & 0 & 0 & 2 & 0 & 0 & 0 \\
\hline 9 & 1 & 0 & 0 & 0 & 0 & 0 & 0 & 2 & 0 & 0 & 2 & 2 & 0 & 0 & 0 & 2 & 2 & 0 & 0 & 0 \\
\hline 10 & 1 & 0 & 0 & 0 & 0 & 0 & 2 & 2 & 0 & 2 & 0 & 2 & 0 & 0 & 2 & 0 & 2 & 0 & 0 & 2 \\
\hline 11 & 1 & 0 & 0 & 0 & 0 & 2 & 0 & 2 & 2 & 2 & 2 & 2 & 0 & 2 & 2 & 2 & 2 & 0 & 2 & 2 \\
\hline 12 & 1 & 0 & 0 & 0 & 2 & 2 & 2 & 0 & 0 & 0 & 0 & 2 & 2 & 0 & 0 & 0 & 2 & 2 & 0 & 2 \\
\hline 13 & 1 & 0 & 0 & 2 & 0 & 0 & 2 & 0 & 0 & 0 & 2 & 0 & 2 & 0 & 0 & 2 & 0 & 2 & 2 & 2 \\
\hline 14 & 1 & 0 & 2 & 2 & 0 & 2 & 2 & 0 & 0 & 2 & 2 & 2 & 2 & 0 & 2 & 2 & 2 & 0 & 0 & 2 \\
\hline 15 & 1 & 2 & 0 & 2 & 2 & 0 & 2 & 0 & 2 & 0 & 0 & 0 & 2 & 2 & 0 & 0 & 2 & 0 & 2 & 0 \\
\hline 16 & 1 & 2 & 2 & 0 & 2 & 2 & 2 & 2 & 2 & 0 & 0 & 2 & 0 & 2 & 0 & 2 & 2 & 2 & 2 & 0 \\
\hline 17 & 1 & 0 & 2 & 2 & 0 & 0 & 0 & 0 & 2 & 0 & 2 & 2 & 2 & 2 & 2 & 0 & 0 & 0 & 2 & 0 \\
\hline 18 & 1 & 2 & 0 & 2 & 0 & 0 & 0 & 2 & 2 & 2 & 0 & 0 & 0 & 0 & 2 & 0 & 0 & 2 & 2 & 0 \\
\hline 19 & 1 & 2 & 2 & 2 & 0 & 0 & 2 & 0 & 0 & 2 & 0 & 0 & 0 & 2 & 2 & 0 & 2 & 0 & 2 & 0 \\
\hline 20 & 1 & 0 & 0 & 2 & 0 & 2 & 2 & 0 & 2 & 2 & 0 & 0 & 2 & 0 & 2 & 2 & 2 & 2 & 2 & 0 \\
\hline
\end{tabular}

show that $G(N)$ does exist for all $N$ that have been checked and is small. Table 2 presents some values. (Similar observations have been made before, cf. pp. 34-35 in [17].)

A rigorous proof of Gilbreath's conjecture appears out of reach, given our knowledge of primes. Maximal gaps between consecutive primes around $x$ are thought to be not much larger than $(\log x)^{2}$. (There is a conjecture of Cramér [5] that these gaps are $O\left((\log x)^{2}\right)$, and numerical evidence [3, 4, 20] supports this conjecture as well as a slightly stronger one of Shanks [16]. There are heuristic arguments, based on work of Maier [9], that suggest the true order of magnitude might be slightly larger, but at most by some fractional power of $\log \log x$.$) However, the best published result is that of Mozzochi [10],$ namely that these gaps are $\leq x^{0.548}$ for large $x$, and even on the assumption of the Riemann Hypothesis the bound for gaps can currently be lowered only to

TABLE 2. Smallest $k$ such that $d_{k}(n)=0,1$, or 2 for all $n<\pi(x)$

\begin{tabular}{c|c|c}
$x$ & $\pi(x)$ & $G(\pi(x))$ \\
\hline $10^{2}$ & 25 & 5 \\
$10^{3}$ & 168 & 15 \\
$10^{4}$ & 1,229 & 35 \\
$10^{5}$ & 9,592 & 65 \\
$10^{6}$ & 78,498 & 95 \\
$10^{7}$ & 664,579 & 135 \\
$10^{8}$ & $5,761,455$ & 175 \\
$10^{9}$ & $50,847,534$ & 248 \\
$10^{10}$ & $455,052,511$ & 329 \\
$10^{11}$ & $4,118,054,813$ & 417 \\
$10^{12}$ & $37,607,912,018$ & 481 \\
$10^{13}$ & $346,065,536,839$ & 635
\end{tabular}


$x^{1 / 2+o(1)}$ as $x \rightarrow \infty$. Further, gaps are thought to behave randomly, but nothing has been proved in this direction. Thus, it is conceivable that for some $n, d_{1}(n)$ is huge, while $d_{1}(j)$ for $1 \leq j \leq n-1$ are small and evenly distributed, so that $d_{2}(n-1), d_{3}(n-2), \ldots, d_{n}(1)$ are all large.

Section 2 discusses some heuristics which indicate that Gilbreath's conjecture is likely to be true. They also indicate what results might suffice to prove this conjecture, and support the suggestion that corresponding conjectures are likely to hold for many other sequences.

\section{Heuristics}

The Gilbreath conjecture appears difficult because there are few tools for studying absolute values of differences of a sequence. We will argue that this difficulty is not serious, and that a proof of Gilbreath's conjecture could probably be obtained if we could prove results about primes that appear much simpler. Unfortunately, those results are also unreachable with known tools.

The $d_{k}(n)$ for $k \geq 1, n \geq 2$, are even, and so $d_{k}(n) \equiv 0$ or $2(\bmod 4)$ in that range. We note that the definition (1.1) then implies that

$$
d_{k+1}(n) \equiv d_{k}(n)+d_{k}(n+1)(\bmod 4)
$$

for $k \geq 1, n \geq 2$. This converts a problem defined by absolute values into one involving linear congruences modulo 4 . Further, these congruences are of the simplest possible type, those that occur in Pascal's triangle modulo 2. (Modulus 4 arises only because of the factor 2 that is present in the $d_{k}(n)$.) If the primes are truly as random as they appear, the $d_{1}(n)$ modulo 4 behave randomly, and then the linear congruence (2.2) suggests that the $d_{k}(n)$ (mod 4) are random. It seems likely, therefore, that about half of the $d_{k}(n)$ will be $0(\bmod 4)$ and half will be $2(\bmod 4)$, with neighboring values almost independent. Numerical evidence supports the conjecture that the $d_{1}(n) / 2$, reduced modulo 2, are asymptotically independent for large $n$. (This is true only asymptotically, though, and among the $d_{1}(n) / 2$ for small $n$, there is an excess of odd ones, so that 2956 of the $d_{1}(n) / 2$ for $2 \leq n \leq 5001$ are odd, for example. Such dependencies among small primes were apparently first noted by F. Roesler and his students in unpublished work about six years ago.) This independence follows also from the Cramér probabilistic model [5], which predicts that gaps between consecutive primes follow the Poisson law. There is a proof by Gallagher [6] that a form of the Poisson law for prime gaps follows from a quantitative form of the Hardy-Littlewood prime $k$-tuple conjecture, and one can apply Gallagher's arguments directly to conclude that the $d_{1}(n) / 2$ reduced modulo 2 are independent for nearby values of $n$. At present, no method is available for proving anything rigorously. If one could prove that the $d_{1}(n)$ modulo 4 are asymptotically independent, the proof might provide enough machinery to show that along any path through which a large $d_{1}(n)$ (which would, after all, have to be $\leq n^{0.55}$ for $n$ large) could possibly produce a large $d_{k}(1)$, there would be enough collisions with nonzero values of $d_{j}(m)$ to reduce the influence of this large difference. (What complicates a possible analysis of this kind is that a large $d_{1}(n)$ would not necessarily produce $d_{n}(1)>1$, but possibly only $d_{m}(1)>1$ for some $m>n$.) 
The arguments above apply to many other sequences in which the first element is a 1, the others even, and where the gaps between consecutive elements are not too large and are sufficiently random. Therefore, as has been observed before (cf. Problem A10 of [7]), there is probably not too much special about the primes in Gilbreath's conjecture.

\section{Computational Results}

Table 2 shows the value of $G(N)$ for various $N$ up to $N=\pi\left(10^{13}\right)$. The function $G(N)$ describes the extreme behavior that is observed. To consider the average behavior, let $g(n)$ for $n \geq 2$ be the least $k$ such that $d_{j}(n)=0$ or 2 for $k \leq j \leq k+1000$. (It is likely that for all $n$ that were computed, $d_{j}(n)=0$ or 2 for all $j \geq g(n)$, but we cannot prove that. If true, it would imply Gilbreath's conjecture.) The average value of $g(n)$ (derived from sampling over intervals of length $8 \times 10^{7}$ ) is presented in Table 3 for several values of $n$. The values of $g(n)$ are considerably smaller than $G(N)$, and this was used to increase the speed of the algorithm, as is noted in $\S 4$.

TABLE 3. Average values of $g(n)$ for $n$ near $N$

\begin{tabular}{c|c}
$N$ & Average of $g(n)$ \\
\hline $10^{8}$ & 22.1 \\
$10^{10}$ & 27.0 \\
$10^{12}$ & 32.8
\end{tabular}

TABLE 4. Average values of $d_{k}(n)$ for various $k$, averaged over 289182 values of $n$ near $\pi\left(10^{12}\right)$

\begin{tabular}{r|c}
$k$ & average of $d_{k}(n)$ \\
\hline 1 & 27.66 \\
2 & 25.51 \\
3 & 19.63 \\
4 & 19.69 \\
5 & 13.50 \\
& \\
10 & 8.33 \\
20 & 4.93 \\
50 & 1.76 \\
100 & 1.15 \\
150 & 1.01
\end{tabular}

Figures $1-3$ show graphs of $d_{k}(n)$ for $\pi\left(10^{12}\right)+1 \leq n \leq \pi\left(10^{12}\right)+10^{4}$ for $k=1,50$, and 100 . This interval appears to be typical, and the graphs show that the process quickly reduces almost all entries to 0 and 2 (the two heavy lines in Figures 2 and 3), with only a few large values that require many additional iterations to reduce. Table 4 shows average values of $d_{k}(n)$ for $\left.\pi\left(10^{12}\right)+1 \leq n \leq \pi\left(10^{12}\right)+\pi\left(8 \times 10^{6}\right)=\pi\left(10^{12}\right)\right)+289182$ and various 


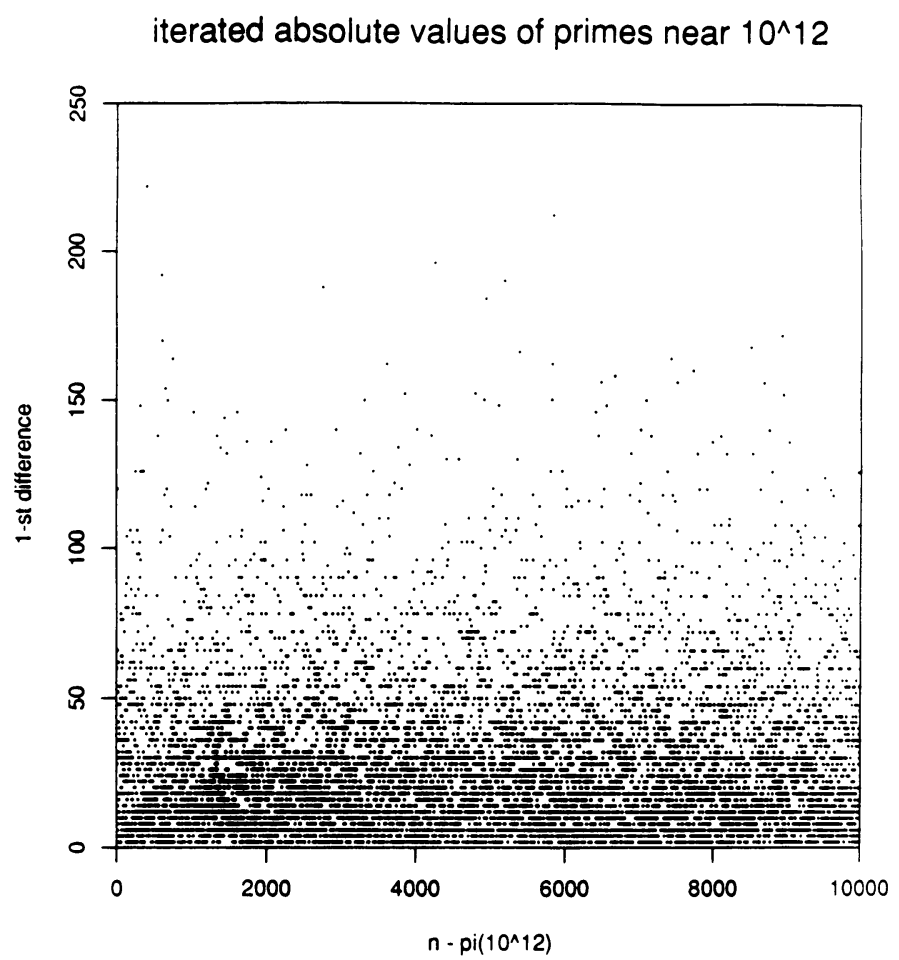

FIGURE 1. Differences $d_{1}(n)$ for $10^{4}$ primes near $10^{12}$ iterated absolute values of primes near $10^{\wedge} 12$

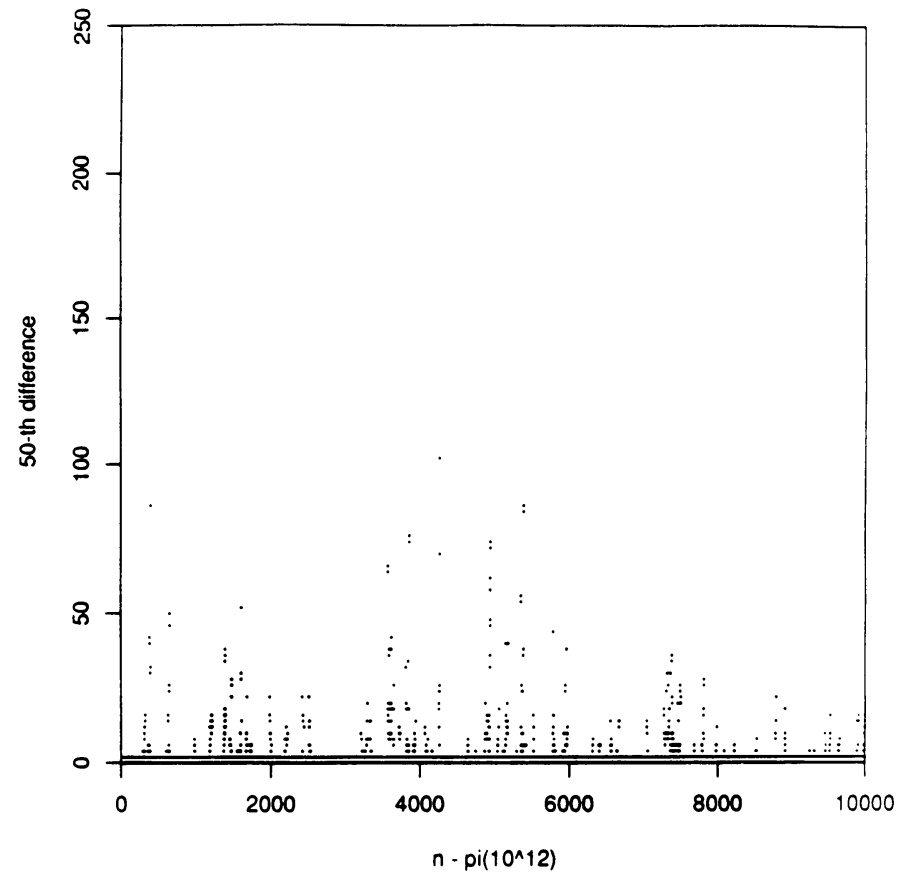

Figure 2. Differences $d_{50}(n)$ for $10^{4}$ primes near $10^{12}$ 


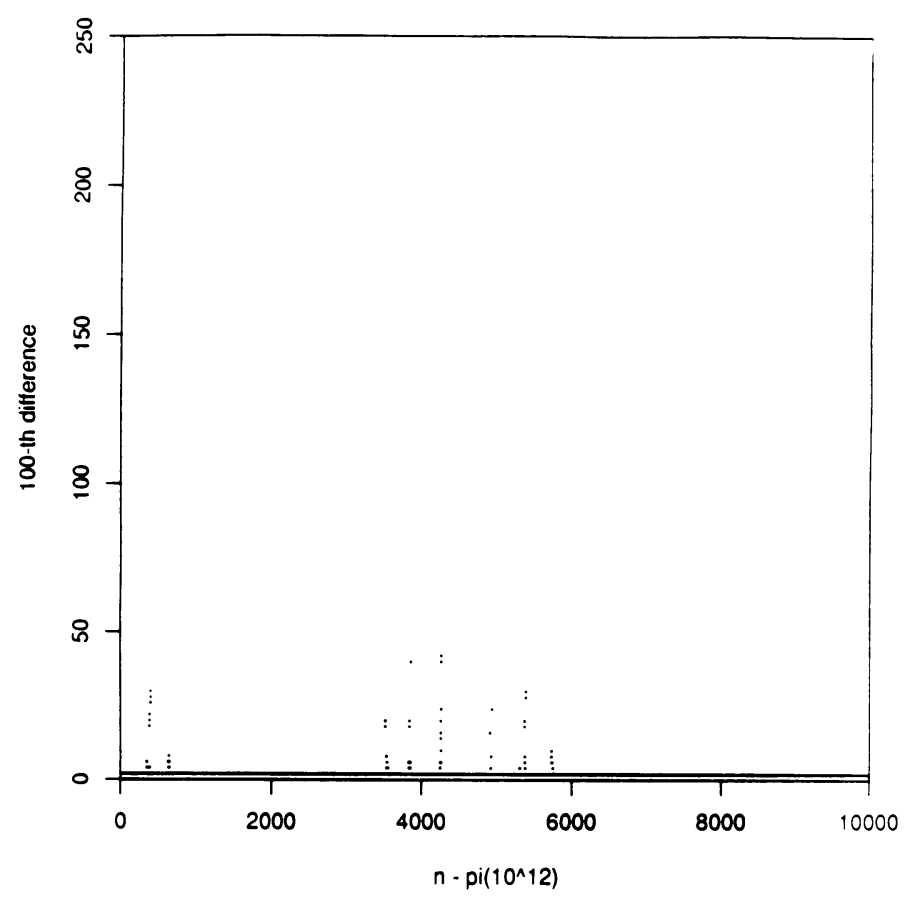

FIGURE 3. Differences $d_{100}(n)$ for $10^{4}$ primes near $10^{12}$

values of $k$. In this set, $d_{k}(n)$ is reduced to 0 and 2 for $k=213$. We note that the average values of $d_{k}(n)$ are not monotone decreasing with $k$.

At the end of the Introduction, we noted that if huge gaps between consecutive primes existed, then Gilbreath's conjecture might be false. Large values of $g(n)$ (and therefore of $G(N)$ ) do appear to be associated with large prime gaps. Every large value of $g(n)$ that was looked at carefully (a very small selection, it should be stressed) was due to a large prime gap. There were some large prime gaps that did not lead to large values of $g(n)$, since other prime gaps nearby served to reduce it. Statistically, though, the association between large prime gaps and large values of $g(n)$ is noticeable. Over $10^{4}$ blocks of length $8 \times 10^{6}$ each around $5.25 \times 10^{12}$ (see $\S 4$ for a definition), the correlation between maximal values of $g(n)$ and maximal prime gaps in a block was 0.52 . The largest value of $g(n)$ that was found, $g(n)=635$ for $n$ close to $\pi\left(7.17716 \times 10^{12}\right)$, is due to the prime gap 674 , which is the largest one up to that height $([3,20])$. The second largest value of $g(n), g(n)=589$ for $n$ close to $\pi\left(2.61494 \times 10^{12}\right)$, is due to the prime gap 652 , which is also the largest one up to its height.

To provide some information about large primes, computations were also done with a set of primes near $10^{50}$ that had been computed by the author earlier for a different purpose. Let $u_{0}(1)<u_{0}(2)<\cdots<u_{0}(8737)$ be the primes in the interval $\left[10^{50}, 10^{50}+10^{6}\right]$, and let $u_{k+1}(n)=\left|u_{k}(n)-u_{k}(n+1)\right|$ for $k \geq 0, n+k \leq 8737$. It was found that $\max _{1 \leq n \leq 8736} u_{1}(n)=920$, and 
$u_{436}(n)=0$ or 2 for all $1 \leq n \leq 8301$, so that 436 iterations sufficed to reduce this finite sequence.

The interval $\left[10^{100}, 10^{100}+10^{7}\right]$ was also tested. Deterministic primality tests would have required too much time, so this interval was first sieved by small primes, and then the remaining elements were tested using the probable prime tests in the Maple symbolic manipulation system. Thus, although the 43271 integers that were found to be all the probable primes are likely to be prime, this has not been rigorously established. The largest gaps between any two consecutive probable primes was 2592 , and 1417 iterations were required to reduce the iterated differences to 0 's and 2's.

\section{Algorithms}

Computation was performed in blocks $B=\left[b_{1}, b_{2}\right]$ of integers from $b_{1}$ (which was always even) to $b_{2}$ (which was odd), with the length of the block varying between $5 \times 10^{5}$ and $8 \times 10^{6}$. The first step was to compute the primes in $B$ using a previously computed table of primes $\leq b_{2}^{1 / 2}$. There is an extensive literature on prime number sieves (for example $[1,2,11,12,13$, $14,18,19])$ and there are some theoretically extremely fast algorithms, such as the Pritchard method [11] that finds all primes $\leq x$ in $O\left(x(\log \log x)^{-1}\right)$ arithmetic operations. However, for numbers of the sizes considered here, the conventional versions of the sieve of Eratosthenes are both faster and simpler to implement (cf. [19]), and so an algorithm similar to the "segmented sieve" of Bays and Hudson [1] and of Brent [3] was used. Since on the processor that was used access to memory was more of a bottleneck than processor speed, some of the operations were segmented further so as to fit into the cache memory.

Once the primes in a block $B$ were determined, they were used together with the last 1000 primes from the preceding block to carry out the second phase, the absolute value of difference iterations. (The number 1000 was chosen to exceed anticipated values of $G(N)$.) Between 50 and 75 iterations were performed on the whole array, and this sufficed to reduce most entries to 0's and 2's. This procedure took most of the computing time of the second phase. Finally, the remaining values of $d_{k}(n)>2$ were grouped into sets that were isolated from each other, and further iterations were performed on those sets. The block $B=\left[10^{12}, 10^{12}+8 \times 10^{6}-1\right]$, which appears to be rather typical, contained 289,182 primes. After 75 iterations, there were 4640 values of $n$ with $d_{75}(n)>$ 2 , and they were grouped into 516 sets which together with the 0 and 2 entries that were associated covered 18,698 values of $n$. (Enough of the adjoining 0 and 2 entries were included to ensure validity of the computation.) This procedure substantially improved the performance of the algorithm. Further speedups can be obtained by more sophisticated versions of this strategy.

Programs were written in Fortran. They were run on a Silicon Graphics 4D220 computer that has 4 R3000 MIPS Computers $25 \mathrm{MHz}$ processors, each rated at about 18 mips, and 128 Mbytes of main memory. (The memory that was required for the programs varied between 5 and 20 megabytes, so was not a constraint.) Programs were run at a low priority so as to use only spare cycles. The code was not carefully optimized, and a speedup by a factor of 2 can probably be achieved. Total run time (for a single processor) was several months, with about 2 seconds necessary to process an interval of length $10^{6}$. About half 
the machine time was spent in sieving for primes, and half in computing the iterated absolute values of the differences.

There is some question about the reliability of a computation as long as this one, especially since there are no easy methods for checking the results. The results stated in the text and in the tables are thought to be correct, but cannot be fully guaranteed. All instances where a value of $g(n)>500$ was found were checked, and one error was discovered that way. The block $B=[M, M+8 \times$ $10^{6}$ ) for $M=8.972168 \times 10^{12}$ had been processed in two separate runs which gave maximal values of $g(n)$ for $n \in B$ of 914 and 261, respectively, with the higher value supposedly due to a prime gap of 1158 . There is no such gap at this height, and 261 is the correct value, as additional computations showed. It was not possible to find out what caused the error.

\section{BIBLIOGRAPHY}

1. C. Bays and R. H. Hudson, The segmented sieve of Eratosthenes and primes in arithmetic progressions to $10^{12}$, BIT 17 (1977), 121-127.

2. S. A. Bengelloun, An incremental primal sieve, Acta Inform. 23 (1986), 119-125.

3. R. P. Brent, The first occurrence of large gaps between successive primes, Math. Comp. 27 (1973), 959-963.

4. __ The first occurrence of certain large prime gaps, Math. Comp. 35 (1980), 1435-1436.

5. H. Cramér, On the order of magnitude of the difference between consecutive prime numbers, Acta Arith. 2 (1937), 23-46.

6. P. X. Gallagher, On the distribution of primes in short intervals, Mathematika 23 (1976), 4-9.

7. R. K. Guy, Unsolved problems in number theory, Springer-Verlag, Berlin and New York, 1981.

8. R. B. Killgrove and K. E. Ralston, On a conjecture concerning the primes, Math. Comp. (Math. Tables Aids Comp.) 13 (1959), 121-122.

9. H. Maier, Primes in short intervals, Michigan Math. J. 32 (1985), 221-225.

10. C. J. Mozzochi, On the difference between consecutive primes, J. Number Theory 24 (1986), 181-187.

11. P. Pritchard, A sublinear additive sieve for finding prime numbers, Comm. ACM 24 (1981), 18-23.

12. __ Explaining the wheel sieve, Acta Inform. 17 (1982), 477-485.

13. __ Fast compact prime number sieves (among others), J. Algorithms 4 (1983), 332-344.

14. $\_$Linear prime-number sieves; A family tree, Sci. Comput. Programming 9 (1987), 17-35.

15. F. Proth, Sur la série des nombres premiers, Nouvelle Correspondance Mathématique 4 (1878), 236-240.

16. D. Shanks, On maximal gaps between successive primes, Math. Comp. 18 (1964), 646-651.

17. W. Sierpinski, A selection of problems in the theory of numbers, Pergamon Press, Oxford, 1964.

18. J. Sorenson, An introduction to prime number sieves, Computer Science Technical Report \#909, Univ. of Wisconsin-Madison, Jan. 1990.

19. An analysis of two prime number sieves, Computer Science Technical Report \#1028, Univ. of Wisconsin-Madison, June 1991.

20. J. Young and A. Potler, First occurrence prime gaps, Math. Comp. 52 (1989), 221-224.

AT\&T Bell Laboratories, Murray Hill, New Jersey 07974

E-mail address: amo@research.att.com 